\title{
PRODUCTS OF $k$-FIBONACCI NUMBERS WHICH ARE REP-DIGITS
}

\author{
ADEL ALAHMADI, ALAA ALTASSAN, FLORIAN LUCA, AND HATOON SHOAIB
}

\begin{abstract}
We show that there are no examples of non-trivial products of consecutive $k$-Fibonacci numbers that are rep-digits.
\end{abstract}

\section{INTRODUCTION}

In 9, F. Luca found all Fibonacci numbers which are rep-digits in base 10. That is, all the solutions of the equation

$$
F_{n}=d\left(\frac{10^{m}-1}{9}\right), \quad d \in\{1,2, \ldots, 9\}
$$

where $\left\{F_{n}\right\}_{n \geq 0}$ is the sequence of Fibonacci numbers given by $F_{0}=0, F_{1}=1$ and $F_{n+2}=F_{n+1}+F_{n}$ for all $n \geq 0$. The largest solution is $F_{10}=55$. Since then this result was generalised and extended in various directions. For example, in [1, we found all Fibonacci numbers which are concatenations of two rep-digits. In [14, Marques and Togbé showed that by imposing that a product of one or more consecutive Fibonacci numbers being a rep-digit with at least two digits, one does not get any extra interesting solutions. Namely, if

$$
F_{n} F_{n+1} \cdots F_{n+\ell-1}=d\left(\frac{10^{m}-1}{9}\right), \quad d \in\{1,2, \ldots, 9\},
$$

for positive integers $\ell, m, n$ with $m \geq 2$, then $\ell=1$ and $n=10$. This problem was also investigated for the sequence of $k$-generalised Fibonacci numbers $\left\{F_{n}^{(k)}\right\}_{n \geq-(k-2)}$ which is given by the recurrence $F_{n+k}^{(k)}=F_{n+k-1}^{(k)}+\cdots+F_{n}^{(k)}$ for all $n \geq-(k-2)$ with initial values $0,0, \ldots, 0,1$ (with $k-1$ consecutive zeros), where the value of 1 corresponds to $n=1$; namely, $F_{1}^{(k)}=1$, and the remaining zeros are in the past $F_{j}^{(k)}=0$ for $j \in\{-(k-2),-(k-3), \ldots, 0\}$. For example, when $k=2$ this coincides with the Fibonacci sequence while when $k=3$ this sequence is also known as the sequence of Tribonacci numbers. In [3], the authors have shown that if

$$
F_{n}^{(3)} F_{n+1}^{(3)} \cdots F_{n+\ell-1}^{(3)}=d\left(\frac{10^{m}-1}{9}\right), \quad d \in\{1,2, \ldots, 9\}
$$

with $m \geq 2$, then $\ell=1$ and $n=8$; that is, $F_{8}^{(3)}=44$. The solution when only one Tribonacci number is involved had been found earlier by Marques in [12]. In the

Date: February 12, 2020.

2010 Mathematics Subject Classification. 11A25, 11B39, 11J86.

Key words and phrases. $k$-Fibonacci numbers, repdigits, linear forms in complex logarithms. 
same paper, Marques conjectured that this is the solution with the largest $k$ of the Diophantine equation

$$
F_{n}^{(k)}=d\left(\frac{10^{m}-1}{9}\right), \quad d \in\{1,2, \ldots, 9\},
$$

in positive integer unknowns $k, n, m$ again with $m \geq 2$. That is, the only solutions of the above Diophantine equation with $m \geq 2$ are $(k, n)=(2,10),(3,8)$ both of which have $m=2$. This was confirmed in [4. In this paper, we bring together these problems and look at products of consecutive $k$-generalised Fibonacci numbers being a rep-digit. Our result is the following theorem.

Theorem 1.1. The only solutions of the Diophantine equation

$$
F_{n}^{(k)} F_{n+1}^{(k)} \cdots F_{n+\ell-1}^{(k)}=d\left(\frac{10^{m}-1}{9}\right), \quad d \in\{1,2, \ldots, 9\}
$$

with $m \geq 2$ are $(k, \ell, n)=(2,1,10),(3,1,8)$.

That is, by imposing that a product of one or more consecutive numbers of the $k$-generalised Fibonacci sequence is a rep-digit with at least 2 digits, we get no additional solutions from the ones we already knew about with $k=2,3$.

\section{Preliminaries}

In this section, we collect some facts about $k$-generalised Fibonacci numbers, algebraic number theory and the theory of linear forms in logarithms of algebraic numbers. One checks easily that the first $k+1$ nonzero terms in $F_{n}^{(k)}$ are powers of 2 , namely

$$
F_{1}^{(k)}=1, \quad F_{2}^{(k)}=1, \quad F_{3}^{(k)}=2, \quad F_{4}^{(k)}=4, \ldots, \quad F_{k+1}^{(k)}=2^{k-1} .
$$

Furthermore, the next two terms are $F_{k+2}^{(k)}=2^{k}-1$ and $F_{k+3}^{(k)}=2^{k+1}-3$ which are odd. We start with the following lemma.

Lemma 2.1. The period of the $k$-generalised Fibonacci sequence modulo 2 is $k+1$. Furthermore, in a period of length $k+1$ there are exactly two $1 s$ and $k-1$ zeros.

Proof. For the first $k+1$ values of $n$, namely $n \in\{-(k-2),-(k-1), \ldots, 1,2\}$, the $k$-generalised Fibonacci numbers are $0,0, \ldots, 0,1,1$ and the next $k+1$ terms of the $k$-generalised Fibonacci sequence modulo 2 for $n \in\{3,4, \ldots, k+3\}$ are also $0,0, \ldots, 0,1,1$, which finishes the proof of both statements because of the recurrence of order $k$.

Let us recall some known facts about the characteristic polynomial and Binet formula for the $k$-generalised Fibonacci numbers. It is known that the characteristic polynomial of the $k$-generalised Fibonacci numbers $F^{(k)}:=\left\{F_{n}^{(k)}\right\}_{n \geq-(k-2)}$, namely

$$
\Psi_{k}(x):=x^{k}-x^{k-1}-\cdots-x-1,
$$

is irreducible over $\mathbb{Q}[x]$ and has just one root outside the unit circle. Let $\alpha:=\alpha(k)$ denote that single root, which is located between $2\left(1-2^{-k}\right)$ and 2 (see [6]). This is called the dominant root of $F^{(k)}$. To simplify notation, in our application we shall omit the dependence of $\alpha$ on $k$. We shall use $\alpha^{(1)}, \ldots, \alpha^{(k)}$ for all roots of $\Psi_{k}(x)$ with the convention that $\alpha^{(1)}:=\alpha$. 
We now consider, for an integer $k \geq 2$, the function

$$
f_{k}(z)=\frac{z-1}{2+(k+1)(z-2)} \quad \text { for } \quad z \in \mathbb{C} .
$$

With this notation, Dresden and Du presented in [6] the following "Binet-like" formula for the terms of $F^{(k)}$ :

$$
F_{n}^{(k)}=\sum_{i=1}^{k} f_{k}\left(\alpha^{(i)}\right) \alpha^{(i)^{n-1}} .
$$

It was proved in [6] that the contribution of the roots which are inside the unit circle to the formula (3) is very small, namely that the approximation

$$
\left|F_{n}^{(k)}-f_{k}(\alpha) \alpha^{n-1}\right|<\frac{1}{2} \text { holds for all } n \geq-(k-2) .
$$

It was proved by Bravo and Luca in 4 that

$$
\alpha^{n-2} \leq F_{n}^{(k)} \leq \alpha^{n-1} \quad \text { holds for all } \quad n \geq 1 \text { and } k \geq 2 .
$$

We continue with some notations and terminologies from algebraic number theory.

Let $\eta$ be an algebraic number of degree $d$ with minimal primitive polynomial over the integers

$$
a_{0} x^{d}+a_{1} x^{d-1}+\cdots+a_{d}=a_{0} \prod_{i=1}^{d}\left(x-\eta^{(i)}\right),
$$

where the leading coefficient $a_{0}$ is positive and the $\eta^{(i)} \mathrm{s}$ are the conjugates of $\eta$. Then the logarithmic height of $\eta$ is given by

$$
h(\eta):=\frac{1}{d}\left(\log a_{0}+\sum_{i=1}^{d} \log \left(\max \left\{\left|\eta^{(i)}\right|, 1\right\}\right)\right) .
$$

In particular, if $\eta=p / q$ is a rational number with $\operatorname{gcd}(p, q)=1$ and $q>0$, then $h(\eta)=\log \max \{|p|, q\}$. The following are some of the properties of the logarithmic height function $h(\cdot)$, which will be used in the next section of this paper without reference:

$$
\begin{aligned}
h\left(\eta \gamma^{ \pm 1}\right) & \leq h(\eta)+h(\gamma), \\
h\left(\eta^{s}\right) & =|s| h(\eta) \quad(s \in \mathbb{Z}) .
\end{aligned}
$$

We next present some useful lemmas that will be used in the next section on this paper. The following lemma was proved by Bravo and Luca in [4].

Lemma 2.2. Let $k \geq 2$, $\alpha$ be the dominant root of $\left\{F_{n}^{(k)}\right\}_{n \geq(k-2)}$, and consider the function $f_{k}(z)$ defined in (2).

(i) The inequalities

$$
\frac{1}{2}<f_{k}(\alpha)<\frac{3}{4}, \quad \text { and } \quad\left|f_{k}\left(\alpha^{(i)}\right)\right|<1, \quad 2 \leq i \leq k
$$

hold.

(ii) The logarithmic height of $f_{k}(\alpha)$ satisfies $h\left(f_{k}(\alpha)\right)<3 \log k$.

The following lemma was proved in $[5]$. 
Lemma 2.3. For $1 \leq n<2^{k / 2}$ and $k \geq 10$, we have

$$
F_{n}^{(k)}=2^{n-2}(1+\zeta), \quad \text { where }|\zeta|<\frac{5}{2^{k / 2}} .
$$

In order to prove our main result Theorem 1.1, we need to use a couple of times a Baker-type lower bound for a nonzero linear form in logarithms of algebraic numbers. There are many such bounds the literature like that of Baker and Wüstholz from [2. We use the one of Matveev from [15]. Matveev proved the following theorem, which is one of our main tools in this paper.

Theorem 2.1 (Matveev's theorem). Let $\gamma_{1}, \ldots, \gamma_{t}$ be positive real algebraic numbers in a real algebraic number field $\mathbb{K}$ of degree $D, b_{1}, \ldots, b_{t}$ be nonzero integers, and assume that

$$
\Lambda:=\gamma_{1}^{b_{1}} \cdots \gamma_{t}^{b_{t}}-1
$$

is nonzero. Then

$$
\log |\Lambda|>-1.4 \times 30^{t+3} \times t^{4.5} \times D^{2}(1+\log D)(1+\log B) A_{1} \cdots A_{t},
$$

where

and

$$
B \geq \max \left\{\left|b_{1}\right|, \ldots,\left|b_{t}\right|\right\}
$$

$$
A_{i} \geq \max \left\{D h\left(\gamma_{i}\right),\left|\log \gamma_{i}\right|, 0.16\right\}, \quad \text { for all } \quad i=1, \ldots, t .
$$

During the course of our calculations, we get some upper bounds on our variables which are too large, thus we need to reduce them. To do so, we use some results from the theory of continued fractions. Specifically, for a nonhomogeneous linear form in two integer variables, we use a slight variation of a result due to Dujella and Pethő (see [7], Lemma 5a). For a real number $X$, we write $\|X\|:=\min \{|X-n|: n \in \mathbb{Z}\}$ for the distance from $X$ to the nearest integer.

Lemma 2.4. Let $M$ be a positive integer, $p / q$ be a convergent of the continued fraction of the irrational number $\tau$ such that $q>6 M$, and $A, B, \mu$ be some real numbers with $A>0$ and $B>1$. If $\varepsilon:=\|\mu q\|-M\|\tau q\|>0$, then there is no solution to the inequality

$$
0<|u \tau-v+\mu|<A B^{-w}
$$

in positive integers $u, v$ and $w$ with

$$
u \leq M \quad \text { and } \quad w \geq \frac{\log (A q / \varepsilon)}{\log B} .
$$

The above lemma cannot be applied when $\mu=0$ (since then $\varepsilon<0$ ). In this case, we use the following criterion of Legendre (see Theorem 8.2.4 and top of page 287 in [16]).

Lemma 2.5 (Legendre). Let $\tau$ be a real number and $x, y$ be integers such that

$$
\left|\tau-\frac{x}{y}\right|<\frac{1}{2 y^{2}}
$$

Then $x / y=p_{k} / q_{k}$ is a convergent of $\tau$. Furthermore,

$$
\left|\tau-\frac{x}{y}\right| \geq \frac{1}{\left(a_{k+1}+2\right) y^{2}} .
$$




\section{The PRoOF of TheOREM 1.1}

3.1. Bounding $\ell$. Assume that $(k, \ell, n, m)$ is a solution of equation (1) with $m \geq 2$. We may also assume that $k \geq 4$ and that $\ell \geq 2$, since the remaining cases have been treated elsewhere. We start with the following observation.

Lemma 3.1. We have $\ell \leq 7$ for $k=4$ and $\ell \leq 5$ for $k \geq 5$.

Proof. For the proof, we use the exponent of 2 in both sides of equation (1). For an integer $s$, let $\nu_{2}(s)$ be the exponent of 2 in the factorisation of $s$. In the right-hand side of equation (1), we have

$$
\nu_{2}\left(d\left(\frac{10^{m}-1}{9}\right)\right)=\nu_{2}\left(d\left(10^{m-1}+\cdots+10+1\right)\right)=\nu_{2}(d) \leq 3 .
$$

Thus, the exponent of 2 in the left-hand side of equation (1) is also at most 3 . Suppose that $k \geq 5$ and $\ell \geq 6$. Since the period of the $k$-generalised Fibonacci sequence modulo 2 is $k+1 \geq 6$, and in any period the sequence modulo 2 has two consecutive 1 and $k-1$ zeros, it follows that among the first $6 k$-generalised Fibonacci numbers $F_{n+j}^{(k)}$ for $j \in\{0,1, \ldots, 5\}$ in the left-hand side of (1), at most two of them are odd and the rest (at least 4 of them) are even. This shows that the left-hand side of equation (1) is a multiple of 16 , a contradiction. A similar argument proves that $\ell \leq 7$ when $k=4$.

3.2. Bounding $n$ in terms of $k$. Next we find an upper bound for $n$ in terms of $k$. We assume that $n \geq 100$. Combining (1) and (5), we get

$$
\begin{aligned}
10^{m-1} & \leq d\left(\frac{10^{m}-1}{9}\right)=F_{n}^{(k)} F_{n+1}^{(k)} \cdots F_{n+\ell-1}^{(k)} \\
& <\alpha^{n-1} \cdot \alpha^{n} \cdots \alpha^{n+\ell-2}=\alpha^{\ell(n-1)+\frac{\ell(\ell-1)}{2}} .
\end{aligned}
$$

Since $\alpha<10$, we get

$$
m \leq \ell(n-1)+\ell(\ell-1) / 2 .
$$

Now, by estimate (4), it follows that we can write

$$
F_{m}^{(k)}=f_{k}(\alpha) \alpha^{m-1}+e_{m}, \quad \text { where } \quad\left|e_{m}\right|<1 / 2
$$

for all $m \geq-(k-2)$. Using this for $m=n, n+1, \ldots, n+\ell-1$ in (1), and putting $a:=f_{k}(\alpha)$ for simplicity, we get

$$
\begin{aligned}
F_{n}^{(k)} \cdots F_{n+\ell-1}^{(k)} & =\left(a \alpha^{n-1}+e_{n}\right) \cdots\left(a \alpha^{n+\ell-2}+e_{n+\ell-1}\right) \\
& =a^{\ell} \alpha^{\ell(n-1)+\ell(\ell-1) / 2}+r(a, \alpha, n, \ell),
\end{aligned}
$$

where $r(a, \alpha, n, \ell)$ involves the part of the expansion of the previous line that contains the product of powers of $a, \alpha$ and at least one of the errors $e_{i}$, for $i=n, \ldots, n+\ell-1$. Thus, $r(a, \alpha, n, \ell)$ is the sum of at most $2^{\ell}-1 \leq 127$ terms with maximum absolute value at most $\left(a^{\ell-1} \alpha^{\ell(n-1)+\ell(\ell-1) / 2}\right) /\left(2 \alpha^{n-1}\right)$. Indeed this follows because $\ell \leq 7$ (see Lemma3.1) and $a>1 / 2>\left|e_{j}\right|$ for $j \in\{n, n+1, \ldots, n+\ell-1\}$ (see Lemma 2.2 (i)).

Combining the above equality with (1), we obtain

$$
a^{\ell} \alpha^{\ell(n-1)+\ell(\ell-1) / 2}-\frac{d}{9} 10^{m}=-\frac{d}{9}-r(a, \alpha, n, \ell) .
$$


Dividing both sides of the above equality by $a^{\ell} \alpha^{\ell(n-1)+\ell(\ell-1) / 2}$ and taking the absolute value, we conclude that

$$
\begin{aligned}
\left|\left(\frac{d}{9 a^{\ell}}\right) \alpha^{-(\ell(n-1)+\ell(\ell-1) / 2)} 10^{m}-1\right| & \leq\left(\frac{d}{9}+|r(a, \alpha, n, \ell)|\right) \cdot a^{-\ell} \alpha^{-(\ell(n-1)+\ell(\ell-1) / 2)} \\
& <\frac{\left(1+127 a^{\ell-1} \alpha^{\ell(n-1)+\ell(\ell-1) / 2} /\left(2 \alpha^{-n+1}\right)\right)}{a^{\ell} \alpha^{(\ell(n-1)+\ell(\ell-1) / 2)}} \\
& <\frac{1+64 a^{\ell-1} \alpha^{(n-1)(\ell-1)+\ell(\ell-1) / 2}}{a^{\ell} \alpha^{\ell(n-1)+\ell(\ell-1) / 2}} \\
& <\frac{128 a^{\ell-1} \alpha^{(n-1)(\ell-1)+\ell(\ell-1) / 2}}{a^{\ell} \alpha^{\ell(n-1)+\ell(\ell-1) / 2}} \\
& =\frac{128}{a \alpha^{n-1}}<\frac{256}{\alpha^{n-1}} .
\end{aligned}
$$

In the above, we used the fact that

$$
64 a^{\ell-1}>64\left(\frac{1}{2}\right)^{\ell-1} \geq 64\left(\frac{1}{2}\right)^{6}=1,
$$

which follows from Lemma 2.2 (i) and Lemma 3.1. We next use Matveev's theorem to find a lower bound for the left-hand side of (12), with the parameters $t:=3$ and $\left(\gamma_{1}, b_{1}\right):=\left(d /\left(9 a^{\ell}\right), 1\right),\left(\gamma_{2}, b_{2}\right):=(\alpha,-(\ell(n-1)+\ell(\ell-1) / 2)),\left(\gamma_{3}, b_{3}\right):=(10, m)$.

The number field containing $\gamma_{1}, \gamma_{2}, \gamma_{3}$ is $\mathbb{L}:=\mathbb{Q}(\alpha)$, which has degree $D:=k$. We claim that $\Lambda:=\gamma_{1}^{b_{1}} \gamma_{2}^{b_{2}} \gamma_{3}^{b_{3}}-1 \neq 0$. Otherwise, we get

$$
a^{\ell} \alpha^{\ell(n-1)+\ell(\ell-1) / 2}=d \cdot 10^{m} / 9 .
$$

Conjugating the above relation by an automorphism $\sigma$ which sends $\alpha^{(1)}$ to $\alpha^{(i)}$ for some $i \in\{2, \ldots, k\}$, and then taking absolute values on both sides of the resulting equality, we obtain

$$
\left|f_{k}\left(\alpha^{(i)}\right)\right|^{\ell}\left|\alpha^{(i)}\right|^{\ell(n-1)+\ell(\ell-1) / 2}=d \cdot 10^{m} / 9,
$$

which is not possible because the left-hand side is smaller than 1 while the righthand side is larger than 10 (since $m \geq 2$ ). Thus, $\Lambda \neq 0$. Next,

$$
\begin{aligned}
h\left(\gamma_{1}\right) & \leq h(d)+h\left(9 a^{\ell}\right) \leq 2 h(9)+\ell h(a) \leq 2 \log 9+21 \log k<24.2 \log k \\
h\left(\gamma_{2}\right) & =\frac{1}{k} \log \alpha<\frac{\log 2}{k} \\
h\left(\gamma_{3}\right) & =\log 10<2.5 .
\end{aligned}
$$

In the above, we used the fact that $k \geq 4$ in the right-most inequality concerning $h\left(\gamma_{1}\right)$. Thus, we can take $A_{1}:=24.2 k \log k, A_{2}:=2$ and $A_{3}:=2.5 k$. By (11), and since $\ell(n-1)+\ell(\ell-1) / 2 \leq 7 n+21<8 n$, we can take $B:=8 n$. Applying Matveev's theorem, we get a lower bound for $|\Lambda|$, and comparing this with 12 we get

$$
\exp \left(-1.8 \times 10^{13} k^{4}(\log k)^{2}(1+\log (8 n))\right)<\frac{256}{\alpha^{n-1}} .
$$

Taking logarithms in the above inequality, we get

$$
(n-1) \log \alpha-\log 256<1.8 \times 10^{13} k^{4}(\log k)^{2}(1+\log (8 n)) .
$$


Since $\alpha \geq \alpha_{4} \geq 1.92$, we get

$$
\begin{aligned}
n & \leq 1+\frac{1.8 \times 10^{13} k^{4}(\log k)^{2}}{\log (1.92)}+\frac{\log 256}{\log 1.92} \\
& <3 \times 10^{13} k^{4}(\log k)^{2}(1+\log 8 n) .
\end{aligned}
$$

By using the fact that $n \geq 100$, we get

$$
8 n \leq 2.4 \times 10^{14} k^{4}(\log k)^{2}(\log 8 n)\left(1+\frac{1}{\log 8 n}\right)<2.8 \times 10^{14} k^{4}(\log k)^{2}(\log 8 n) .
$$

Thus,

$$
\frac{8 n}{\log 8 n}<2.8 \times 10^{14} k^{4}(\log k)^{2} .
$$

Using the fact that $x / \log x<A$ implies $x<2 A \log A$ for $A \geq 10$ (see Lemma 7 in [8]), $\log k<k$ and $k \geq 4$, we get that

$$
\begin{aligned}
8 n & \leq 5.6 \times 10^{14} k^{4}(\log k)^{2} \log \left(2.8 \times 10^{14} k^{4}(\log k)^{2}\right) \\
& <5.6 \times 10^{14} k^{4}(\log k)^{2}(14 \log 10+\log 2.8+6 \log k) \\
& <5.6 \times 10^{14} k^{4}(\log k)^{3}\left(6+\frac{14 \log 10+\log 2.8}{\log k}\right) \\
& <1.68 \times 10^{16} k^{4}(\log k)^{3} \quad(k \geq 4),
\end{aligned}
$$

which implies that

$$
n<2.1 \times 10^{15} k^{4}(\log k)^{3} .
$$

Thus, we proved the following intermediary result.

Lemma 3.2. If $(k, \ell, n, m)$ is a solution of equation (1) with $n \geq 100$ and $k \geq 4$, then

$$
n<2.1 \times 10^{15} k^{4}(\log k)^{3} .
$$

3.3. Bounding $k$ absolutely. We would like to apply Lemma 2.3 in order to approximate $F_{n}^{(k)}$ as in (7), but for that we need to be in the range $n<2^{k / 2}$. The inequality

$$
2.1 \times 10^{15} k^{4}(\log k)^{3}<2^{k / 2}
$$

holds for all $k \geq 180$. Thus, by Lemma 3.2 the inequality $n<2^{k / 2}$ is satisfied when $k \geq 180$. We work under this assumption in this section and we will cover the range $k<180$ later. Therefore we are in the hypothesis of Lemma 2.3 , so we can write

$$
F_{n+j}^{(k)}=2^{n+j-2}\left(1+\zeta_{j}\right), \quad \text { with } \quad\left|\zeta_{j}\right|<\frac{5}{2^{k / 2}} \quad \text { for } \quad j \in\{0, \ldots, \ell-1\} .
$$

Multiplying the above estimates for $j \in\{0,1, \ldots, \ell-1\}$, we get

$$
\begin{aligned}
F_{n}^{(k)} \cdots F_{n+\ell-1}^{(k)} & =2^{n-2+(n+1-2)+\cdots+(n+\ell-3)} \prod_{j=0}^{\ell-1}\left(1+\zeta_{j}\right) \\
& =: 2^{\ell n+\ell(\ell-5) / 2}(1+\zeta), \quad \text { where } \quad|\zeta|<\frac{70}{2^{k / 2}} .
\end{aligned}
$$


The only fact to justify the above estimate is the upper bound on the absolute value of the error which is denoted by $\zeta$. This follows because

$$
\begin{aligned}
|\zeta| & =\left|\sum_{\substack{I \subset\{0,1, \ldots, \ell-1\} \\
I \neq \emptyset}} \prod_{j \in I} \zeta_{j}\right| \leq \sum_{\substack{I \subset\{0,1, \ldots, \ell-1\} \\
I \neq \emptyset \in \emptyset}} \prod_{\substack{j \in I \\
\mid}}\left|\zeta_{j}\right|<\sum_{\substack{I \subset\{0,1, \ldots, \ell-1\} \\
I \neq \emptyset}} \prod_{j \in I}\left(\frac{5}{2^{k / 2}}\right) \\
& =\left(1+\frac{5}{2^{k / 2}}\right)^{\ell}-1<\exp \left(\frac{5 \ell}{2^{k / 2}}\right)-1 \leq \exp \left(\frac{35}{2^{k / 2}}\right)-1<\frac{70}{2^{k / 2}},
\end{aligned}
$$

where in the last estimate we used the fact that $e^{x}-1<2 x$ for $x \in(0,1 / 2)$, with $x:=35 / 2^{k / 2}$ (note that the inequality $x<1 / 2$ holds for such $x$ because $k$ is large). Thus, we write equation (1) as follows

$$
2^{\ell n+\ell(\ell-5) / 2}(1+\zeta)=d\left(\frac{10^{m}-1}{9}\right) .
$$

Thus,

$$
2^{\ell n+\ell(\ell-5) / 2}-\frac{d 10^{m}}{9}=-2^{\ell n+\ell(\ell-5) / 2} \zeta-\frac{d}{9} .
$$

Dividing both sides by $2^{\ell n+\ell(\ell-5) / 2}$ and taking absolute values, we get

$$
\begin{aligned}
\left|1-\left(\frac{d}{9}\right) 2^{-(\ell n+\ell(\ell-5) / 2)} 10^{m}\right| & \leq\left|\zeta+\frac{d}{9 \times 2^{\ell n+\ell(\ell-5) / 2}}\right|<|\zeta|+\frac{1}{2^{\ell n+\ell(\ell-5) / 2}} \\
& \leq \frac{70}{2^{k / 2}}+\frac{1}{2^{\ell n+\ell(\ell-5) / 2}}<\frac{71}{2^{\min \{k / 2, \ell n+\ell(\ell-5) / 2\}}} .
\end{aligned}
$$

Now let us show that the minimum in the exponent of 2 in the right-hand side of the above estimate is $k / 2$. Indeed, $n \geq k-4$, for if not, then $n \leq k-5$, therefore $n+\ell-1 \leq n+6 \leq k+1$. Thus, the interval $[n, n+\ell-1]$ is contained in the interval $[1, k+1]$, and in this last interval all members of $F^{(k)}$ are powers of 2 . Thus, the left-hand side of (1) is a power of 2 which is a rep-digit with at least two digits $m$, a contradiction. Hence, $n \geq k-4$, so that

$$
\ell n+\ell(\ell-5) / 2 \geq 2(k-4)+2(2-5) / 2=2 k-11>k / 2,
$$

where the last inequality holds because $k \geq 180$. Thus, bound $(14)$ becomes

$$
\left|1-\left(\frac{d}{9}\right) 2^{-(\ell n+\ell(\ell-5) / 2)} 10^{m}\right|<\frac{71}{2^{k / 2}} .
$$

In the left-hand side, we apply Matveev's theorem. We first need to check that the expression is non-zero. But if it were zero, we would get that

$$
\frac{d 10^{m}}{9}=2^{\ell n+\ell(\ell-5) / 2}
$$

which is impossible by unique factorisation since 5 appears with exponent $m \geq 2$ in the left-hand side but not on the right-hand side. So, we can apply Matveev's theorem with the parameters $t:=3$ and

$$
\left(\gamma_{1}, b_{1}\right):=(d / 9,1),\left(\gamma_{2}, b_{2}\right):=(2,-(\ell n+\ell(\ell-5) / 2)),\left(\gamma_{3}, b_{3}\right):=(10, m) .
$$

The number field containing $\gamma_{1}, \gamma_{2}, \gamma_{3}$ is $\mathbb{Q}$ of degree $D:=1$. Next, we can take $A_{1}:=\log 9, A_{2}:=\log 2$ and $A_{3}:=\log 10$. Since $\ell n+\ell(\ell-5) / 2<\ell(n-1)+\ell(\ell-$ $1) / 2 \leq 7 n+21<8 n$ (by the arguments from the previous application of Matveev's theorem in Subsection 3.2 , we can take as previously $B:=8 n$. Applying Matveev's 
theorem, we get a lower bound for the left-hand side of (15), By comparing this bound to 15 , we have

$$
\exp \left(-5.1 \times 10^{11}(1+\log (8 n))\right)<\frac{71}{2^{k / 2}},
$$

which leads to

$$
(k / 2) \log 2-\log 71<5.1 \times 10^{11}(1+\log (8 n)) .
$$

Combining the above inequality with 13 , we get

$$
(k / 2) \log 2-\log 71<5.1 \times 10^{11}\left(1+\log \left(8 \times 2.1 \times 10^{15} k^{4}(\log k)^{3}\right)\right),
$$

which implies $k<3 \times 10^{14}$. Hence, by (13), we have that

$$
n<2.1 \times 10^{15}\left(3 \times 10^{14}\right)^{4}\left(\log \left(3 \times 10^{14}\right)\right)^{3},
$$

so $n<7 \times 10^{77}$. To sum up, we record these bounds in the following lemma.

Lemma 3.3. We have $k<3 \times 10^{14}$ and $n<7 \times 10^{77}$.

3.4. Lowering the bound on $k$. Next we need to lower the upper bound for $k$. For this, we write

$$
\Gamma:=m \log 10-(\ell n+\ell(\ell-5) / 2) \log 2+\log (d / 9),
$$

and note that 15 implies that

$$
\left|e^{\Gamma}-1\right|<\frac{71}{2^{k / 2}} .
$$

Since $k \geq 180$ is large, the right-hand side above is smaller than $1 / 2$, so the above inequality implies that

$$
|\Gamma|<\frac{142}{2^{k / 2}} .
$$

In the above, we used the fact that if $x$ and $y$ are real numbers with $\left|e^{x}-1\right|<y$ and $y \in(0,1 / 2)$, then $|x|<2 y$. Dividing the last inequality above by $\log 2$, we get

$$
|m \tau-N+\mu|<\frac{A}{B^{k}},
$$

where

$$
\tau:=\frac{\log 10}{\log 2}, \mu:=\frac{\log (d / 9)}{\log 2}, A:=205, B:=2^{1 / 2},
$$

and $N:=n \ell+\ell(\ell-5) / 2$. Since $N<8 n$, by Lemma 3.3 , it follows that $N<$ $6 \times 10^{78}:=M$. We need a convergent $p_{j} / q_{j}$ of $\tau$ such that $q_{j}>6 M$. The convergent $p_{164} / q_{164}$ has $q_{164}>7 \times 10^{80}>6 M$. Furthermore, computing $\left\|\mu q_{164}\right\|-M\left\|\tau q_{164}\right\|$ for $d \in\{1, \ldots, 8\}$, we get that $\varepsilon>0.01$. By Lemma 2.4 we get that

$$
k<\frac{\log \left(A q_{164} / 0.01\right)}{\log B}<570 .
$$

Hence, $k<570$. This was if $d \neq 9$. If $d=9$, then $\mu=0$ and we cannot apply Lemma 2.4 as $\varepsilon<0$, so instead we apply Lemma 3.1. Namely, (16) implies

$$
\left|\frac{m}{N}-\frac{1}{\tau}\right|<\frac{A / \tau}{B^{k} N}<\frac{62}{N 2^{k / 2}} .
$$

We need to check that the right hand side above is at most $1 /\left(2 N^{2}\right)$, which is equivalent to

$$
124 N<2^{k / 2} .
$$


Since $N<8 n$, the above inequality 19 is implied, by invoking $(13)$, by the inequality

$$
124 \times 8 \times 2.1 \times 10^{15} k^{4}(\log k)^{3}<2^{k / 2},
$$

which holds for $k \geq 200$. So, assuming $k \geq 200$, we can apply Lemma 3.1. Thus, $m / N=p_{j} / q_{j}$ for some convergent $p_{j} / q_{j}$ of $1 / \tau$ (we keep the previous convergents since the convergents of $1 / \tau$ are, with the exception of the very first one which is $0 / 1$, the same as the converges of $\tau$ ). Since $q_{j} \leq N \leq M<q_{164}$, it follows that $m / N=p_{j} / q_{j}$ for some $j \leq 163$. With Mathematica, we computed that $\max \left\{a_{j}: a \leq j \leq 164\right\}=5393$. Hence, by Lemma 3.1 and 19 , we get

$$
\frac{1}{5395 \times N^{2}}<\frac{62}{N 2^{k / 2}}
$$

so

$$
2^{k / 2} \leq 5395 \times 62 N<5395 \times 62 \times\left(6 \times 10^{78}\right),
$$

which gives $k<570$. Thus, in all cases, we got $k<570$.

We can iterate one more time the same program. Namely, feeding $k<570$ into (13), we get $n<6 \times 10^{28}$, so $N \leq 8 n \leq 5 \times 10^{29}:=M$. We now choose $q:=q_{64}>4 \times 10^{30}>6 \mathrm{M}$. For $d \in\{1, \ldots, 8\}$, we compute that $\varepsilon>0.08$, which gives $k \leq 230$. For $d=9$, the inequality $(20)$ is satisfied for $k \geq 200$. Thus, by the previous argument, we get that $m / N=p_{j} / q_{j}$ for some $j \leq 63$. We now have $\max \left\{a_{k}: k \leq 63\right\}=42$, and the previous argument gives that the analog of (21) is

$$
2^{k / 2} \leq 44 \times 62 N<44 \times 62 \times\left(5 \times 10^{29}\right),
$$

which gives that $k \leq 220$. Hence, $k \leq 230$ holds in all cases. Let us record this information.

Lemma 3.4. We have $k \leq 230$.

3.5. Bounding $n$. By $(13)$ together with the fact that $k \leq 230$, we have $n<10^{27}$, so $N_{1}:=\ell(n-1)+\ell(\ell-1) / 2=\ell n+\ell(\ell-3) / 2<8 n<8 \times 10^{27}:=M$. Assume that $n \geq 100$. Since $\alpha \geq \alpha(4) \geq 1.92$, it follows that the right-hand side of inequality (12) is $<1 / 2$. Putting

$$
\Gamma_{k}:=m \log 10-N_{1} \log \alpha+\log \left(d /\left(9 a^{\ell}\right)\right),
$$

inequality 12 shows that

$$
\left|\Gamma_{1}\right|<\frac{512}{\alpha^{n-1}}
$$

Dividing both sides by $\log \alpha$, we get

$$
\left|m \tau-N_{1}+\mu\right|<\frac{A}{B^{n-1}}, \quad \tau:=\frac{\log 10}{\log \alpha}, \mu:=\frac{\log \left(d /\left(9 a^{\ell}\right)\right)}{\log \alpha}, A:=785, B:=1.92 .
$$

Here, we used the fact that $512 / \log \alpha<512 / \log 1.92<785$. For each $k \in[4,230]$, we generated the convergent $p_{130} / q_{130}$ of $\tau:=\tau_{k}$. The minimum value of $q_{130}$ exceeds $10^{55}$, so they all satisfy $q_{130}>6 M$. Further, their maximum is $<8 \times 10^{78}$. We checked that for all such $k \in[4,230], d \in[1,9], \ell \in[1,7]$ the inequality $\left\|\mu q_{130}\right\|-$ $M\left\|\tau q_{130}\right\|>10^{-7}$ holds. Thus,

$$
n-1<\frac{\log \left(785 \times 8 \times 10^{78} / 10^{-7}\right)}{\log (1.92)},
$$


which implies $n<320$. We now computed the amount $F_{n}^{(k)} \cdots F_{n+\ell-1}^{(k)}\left(\bmod 10^{10}\right)$ for every $k \leq 230$, every $n \leq 350$, and every $\ell \leq 7$. None of these numbers were rep-digits with at most 10 digits. Thus, the theorem is proved.

\section{AcKnowledgements}

This study was funded by King Abdulaziz University, Deanship of Scientific Research (grant number RG-26-130-40).

\section{REFERENCES}

[1] A. Alahmadi, A. Altassan, F. Luca and H. Shoaib, Fibonacci numbers which are concatenations of two repdigits, preprint, 2019.

[2] A. Baker and G. Wüstholz, Logarithmic forms and group varieties, J. Reine Angew. Math. 442 (1993), 19-62.

[3] E. Bravo, C.-A. Gómez and F. Luca, Products of consecutive Tribonacci numbers with only one distinct digit, J. Integer Sequences 22 (2019), A 19.6.3.

[4] J.J. Bravo and F. Luca, On a conjecture about repdigits in k-generalised Fibonacci sequences, Publ. Math. Debrecen 82 (2013), 623-639.

[5] M. Ddamulira and F. Luca, On the problem of Pillai with $k$-generalised Fibonacci numbers and powers of 3, preprint 2019.

[6] G. P. Dresden and Z. Du, A simplified Binet formula for $r$-generalised Fibonacci numbers, Journal of Integer Sequences, 17 (2014): Article 14.4.7, 9pp.

[7] A. Dujella and A. Pethö, A generalization of a theorem of Baker and Davenport, Quart. J. Math. Oxford Ser. (2) 49 (1998), 291-306.

[8] S.S. Gúzman and F. Luca, Linear combinations of factorials and $S$-units in a binary recurrence sequences, Annales Mathemántiques du Québec, 38 (2014), 169-188.

[9] F. Luca, Fibonacci and Lucas numbers with only one distinct digit, Port. Math. 57 (2000), 243-254.

[10] F. Luca, Distinct digits in base b expansions of linear recurrence sequences, Quaest. Math. 23 (2000), 389-404.

[11] F. Luca, Repdigits which are sums of at most three Fibonacci numbers, Math. Comm. 17 (2012), 1-11.

[12] D. Marques, On k-generalized Fibonacci numbers with only one distinct digit, Utilitas Math. 98 (2015), 23-31.

[13] D. Marques and A. Togbé, On terms of linear recurrence sequences with only one distinct block of digits, Colloquium Mathematicum 124 (2011), 145-155.

[14] D. Marques and A. Togbé, On repdigits as product of consecutive Fibonacci numbers, Rend. Istit. Mat. Univ. Trieste, 44 (2012), 393-397.

[15] E. M. Matveev, An explicit lower bound for a homogeneous rational linear form in logarithms of algebraic numbers, II, Izv. Ross. Akad. Nauk Ser. Mat. 64 (2000), 125-180. English translation in Izv. Math. 64 (2000), 1217-1269.

[16] R. M. Murty and J. Esmonde, Problems in algebraic number theory. Second edition. Graduate Texts in Mathematics, 190. Springer-Verlag, New York, 2005. 
Research Group in Algebraic Structures and its Applications, King Abdulaziz UniVERsity, Jeddah, SAudi Arabia

E-mail address: analahmadi@kau.edu.sa

Research Group in Algebraic Structures and its Applications, King Abdulaziz UniVersity, Jeddah, SAudi Arabia

E-mail address: aaltassan@kau.edu.sa

School of Maths, Wits University, South Africa, Max Planck Institute for Mathematics, Bonn, Germany, Research Group in Algebraic Structures and Applications, King Abdulaziz University, Jeddah, Saudi Arabia

E-mail address: florian.luca@wits.ac.za

Research Group in Algebraic Structures and its Applications, King Abdulaziz University, Jeddah, Saudi Arabia

E-mail address: hashoaib@kau.edu.sa 